\title{
Trip Length and Sufficient Number of Alternative Fuel Stations
}

\author{
Masashi Miyagawa*
}

\begin{abstract}
This paper develops a model for determining the sufficient number of stations for alternative fuel vehicles. The model extends a previous model to incorporate both flow demand and the trip length distribution, thereby providing a more appropriate framework for estimating the sufficient number of stations. The service level is represented as the probability that the vehicle can make the repeated round trip between randomly selected origin and destination. The probability is obtained for the random pattern of stations for three cases: fuel is available at both origin and destination, fuel is available at either origin or destination, and fuel is available at neither origin nor destination. The analytical expressions for the probability demonstrate how the number of stations, the trip length, the vehicle range, and the refueling availability at origin and destination affect the service level of stations. The number of stations required to achieve a certain level of service is then calculated.
\end{abstract}

Keywords: Location, Flow demand, Trip length distribution, Vehicle range, Round trip

\section{Introduction}

Alternative fuel vehicles powered by electricity, hydrogen, and biofuels have attracted attention because of environmental, geopolitical, and financial concerns. The transition from gasoline engine vehicles to alternative fuel vehicles, however, would be difficult. One of the most significant factors for the transition is the adequate availability of refueling stations. ${ }^{1)}$

Several approaches have been proposed to calculate the sufficient number of alternative fuel stations. Melaina ${ }^{2)}$ developed three approaches for estimating the number of hydrogen stations based on the number of existing gasoline stations, metropolitan land areas, and lengths of major roads. Melaina and Bremson ${ }^{3)}$ proposed a sufficient level of station coverage that meets the refueling needs of the general population in urban areas. Nicholas et al. ${ }^{4)}$ developed a GIS model for siting hydrogen stations and examined the effect of the number of stations on the average driving time to the nearest station. Nicholas and Ogden $^{5)}$ studied the regional variation in the number of stations needed to achieve a travel time target. Bersani et al. ${ }^{6}$ ) formulated a model for selecting gasoline stations to be converted to hydrogen stations.

Most of the previous studies concerning the sufficient number of stations focused on the distance to the nearest station. That is, drivers are assumed to use the nearest station from their home. Refueling stations are, however, typical flow demand facilities in that demand

*University of Yamanashi 
for service can be expressed as flow. ${ }^{7-9)}$ In fact, drivers usually refuel their vehicles on pre-planned trips from origin to destination. Nicholas ${ }^{10)}$ and Kelley and Kuby ${ }^{11)}$ found that more drivers choose a station on their least deviation route than the station closest to home. This type of refueling behavior should therefore be taken into account when discussing the sufficient number of stations.

In this paper, we develop a model for determining the sufficient number of alternative fuel stations. To incorporate flow demand, the service level is represented as the probability that the vehicle can make the repeated round trip between randomly selected origin and destination. Although Miyagawa ${ }^{12}$ proposed a similar model, we extend the scope by introducing the trip length distribution. Since travel demand depends on the trip length, this extension leads to a more realistic framework for estimating the sufficient number of stations. In addition, we deal with not only stations on a plane but also stations on a line. The model on a line gives an insight into analysis on road networks. ${ }^{13)}$

An efficient location of alternative fuel stations has also been studied. Kuby and $\mathrm{Lim}^{14)}$ developed the flow refueling location model (FRLM) for optimally locating refueling facilities. The FRLM locates $p$ facilities to maximize the total flow volume that can be refueled. A number of applications and extensions of the FRLM have been proposed. ${ }^{15)-19)}$ In these location models, the number of stations to be located is an input. Our model thus supplements location models of alternative fuel stations.

The remainder of this paper is organized as follows. The next section develops a model for determining the sufficient number of alternative fuel stations. Sections 3 and 4 obtain the probability of making the round trip for stations on a line and on a plane, respectively. Section 5, as a numerical example, calculates the sufficient number of stations required to achieve a certain level of service. The final section presents concluding remarks.

\section{Model}

Consider trips using alternative fuel vehicles. Let $r$ be the vehicle range, which is the maximum distance that the vehicle with full tank of fuel can drive. Origins and destinations are selected at random within a study region. Let $t$ be the trip length between origin and destination and $f(t)$ be the probability density function of $t$, which we call the trip length distribution.

Let $P$ be the probability that the vehicle can make the repeated round trip between randomly selected origin and destination. Drivers are assumed to deviate from their shortest paths to refuel their vehicles, irrespective of the deviation distance. Refueling is allowed only once for each one-way trip. The reasons for this refueling assumption are the following. First, short distance trips are more frequent and thus more important than long distance trips with multiple refueling. Note that the range of most of the electric vehicles is shorter than $200 \mathrm{~km}$. Second, if multiple refueling is allowed for short distance trips, the sufficient number of stations decreases but the inconvenience of drivers increases. Since the probability $P$ depends on the refueling availability at origin and destination, three cases are considered: fuel is available at both origin and destination, fuel is available at either origin or destination, and fuel is available at neither origin nor destination.

Refueling stations are assumed to be randomly distributed within the study region. Although the random pattern of stations may seem unrealistic, the random pattern is 
useful in exploratory analysis where no research hypothesis exists. In fact, the random pattern has been used in location analysis. ${ }^{20)-22)}$ The random pattern yields analytical expressions for the probability of making the round trip, and provides fundamental relationships between variables. In addition, when stations are sparse, the pattern of stations makes little impact on the basic properties of the probability of making the round trip. By comparing the probabilities for grid and random patterns of stations, Miyagawa ${ }^{12)}$ demonstrated that the difference between them is relatively small.

\section{Stations on a line}

Suppose that $n$ stations are randomly distributed on a line segment of length $L$. The model on a line can be applied to the analysis on major highways.



(a)

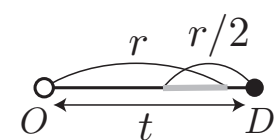

(b)

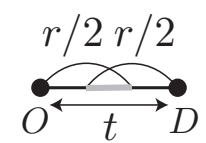

(c)

Figure 1. Calculation of the probability on a line.

First, we assume that fuel is available at both origin $O$ and destination $D$. The vehicle can then start at $O$ with full tank of fuel. If $t \leq r$, the vehicle can reach $D$ without refueling, fill up at $D$, and return to $O$. If $t>2 r$, the vehicle cannot reach $D$ because more than one refueling is needed. If $r<t \leq 2 r$, the vehicle can make the round trip if a station exists within the distance $r$ of both $O$ and $D$. In fact, the vehicle can reach the station, fill up at the station, go to $D$, fill up again at $D$, turn round, fill up again at that same station, and return to $O$. Thus, the probability of making the repeated round trip is the probability that the line segment of length $2 r-t$, as depicted in Fig. 1a, contains at least one station. The probability that a line segment of length $L$ contains exactly $x$ randomly distributed points, denoted by $P(x, L)$, is given by the Poisson distribution as

$$
P(x, L)=\frac{(\rho L)^{x}}{x !} \exp (-\rho L)
$$

where $\rho$ is the density of points. ${ }^{23)}$ The probability of making the repeated round trip for a trip of length $t$, denoted by $p(t)$, is obtained as

$$
\begin{aligned}
p(t) & = \begin{cases}1, & t \leq r, \\
1-P(0,2 r-t), & r<t \leq 2 r, \\
0, & t>2 r,\end{cases} \\
& = \begin{cases}1, & t \leq r, \\
1-\exp \left\{-\frac{n}{L}(2 r-t)\right\}, & r<t \leq 2 r, \\
0, & t>2 r .\end{cases}
\end{aligned}
$$

The probability of making the repeated round trip for all trips is then given by

$$
P=\int_{0}^{2 r} p(t) f(t) \mathrm{d} t
$$


Next, we assume that fuel is available at either origin $O$ or destination $D$. Since the repeated round trip is considered, we can assume that fuel is available at only $O$ without loss of generality. To ensure that the vehicle can make the round trip, the vehicle is required to reach $D$ with at least half a tank remaining. If $t \leq r / 2$, the vehicle can start at $O$ with full tank of fuel, reach $D$, and return to $O$ without running out of fuel. If $t>3 r / 2$, the vehicle cannot make the round trip without refueling more than once. If $r / 2<t \leq 3 r / 2$, the vehicle can make the round trip if a station exists within the distance $r$ of $O$ and within the distance $r / 2$ of $D$. In fact, the vehicle can reach the station, fill up at the station, go to $D$, turn round, fill up again at that same station, and return to $O$. Thus, the line segment of length $3 r / 2-t$, as depicted in Fig. 1b, must contain at least one station. The probability of making the repeated round trip for a trip of length $t$ is obtained as

$$
p(t)= \begin{cases}1, & t \leq \frac{r}{2} \\ 1-\exp \left\{-\frac{n}{L}\left(\frac{3}{2} r-t\right)\right\}, & \frac{r}{2}<t \leq \frac{3}{2} r \\ 0, & t>\frac{3}{2} r .\end{cases}
$$

The probability of making the repeated round trip for all trips is given by

$$
P=\int_{0}^{3 r / 2} p(t) f(t) \mathrm{d} t .
$$

Finally, we assume that fuel is available at neither origin $O$ nor destination $D$. We also assume that the vehicle starts at $O$ with half a tank of fuel and reaches $D$ with at least half a tank remaining, as suggested by Kuby and Lim. ${ }^{14)}$ This assumption ensures that the vehicle can make the repeated round trip. If $t>r$, the vehicle cannot make the round trip without refueling more than once. If $t \leq r$, the vehicle can complete the round trip with at least half a tank remaining if a station exists within the distance $r / 2$ of both $O$ and $D$. In fact, the vehicle can reach the station, fill up at the station, go to $D$, turn round, fill up again at that same station, and return to $O$. Thus, the line segment of length $r-t$, as depicted in Fig. 1c, must contain at least one station. The probability of making the repeated round trip for a trip of length $t$ is obtained as

$$
p(t)= \begin{cases}1-\exp \left\{-\frac{n}{L}(r-t)\right\}, & t \leq r, \\ 0, & t>r .\end{cases}
$$

The probability of making the repeated round trip for all trips is given by

$$
P=\int_{0}^{r} p(t) f(t) \mathrm{d} t
$$

\section{Stations on a plane}

Suppose that $n$ stations are randomly distributed on a region of area $S$. Distance is measured as the Euclidean distance. The model on a plane can be applied to the analysis on regions with a dense road network.

First, we assume that fuel is available at both origin $O$ and destination $D$. If $r<t \leq 2 r$, to make the round trip, the intersection of the two circles centered at $O$ and $D$ with radius 


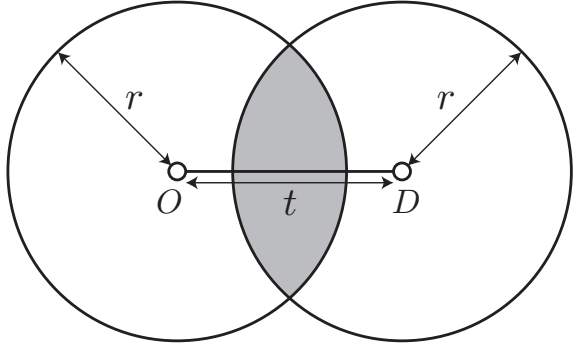

(a)

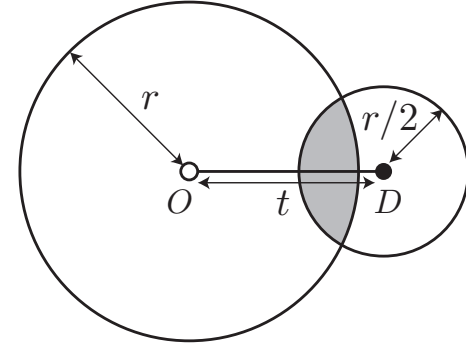

(b)



(c)

Figure 2. Calculation of the probability on a plane.

$r$, as depicted in Fig. 2a, must contain at least one station. The probability of making the repeated round trip for a trip of length $t$ was obtained by Miyagawa ${ }^{12)}$ as

$$
p(t)= \begin{cases}1, & t \leq r \\ 1-\exp \left\{-\frac{n}{S}\left(2 r^{2} \arccos \frac{t}{2 r}-\frac{t}{2} \sqrt{4 r^{2}-t^{2}}\right)\right\}, & r<t \leq 2 r \\ 0, & t>2 r\end{cases}
$$

The probability of making the repeated round trip for all trips is given by Eq. (3).

Next, we assume that fuel is available at only origin $O$. If $r / 2<t \leq 3 r / 2$, to make the round trip, the intersection of the circle centered at $O$ with radius $r$ and the circle centered at $D$ with radius $r / 2$, as depicted in Fig. $2 \mathrm{~b}$, must contain at least one station. The probability of making the repeated round trip for a trip of length $t$ was obtained by Miyagawa $^{12)}$ as

$$
p(t)=\left\{\begin{array}{cl}
1, & t \leq \frac{r}{2}, \\
1-\exp \left[-\frac{n}{S}\left\{r^{2} \arccos \frac{4 t^{2}+3 r^{2}}{8 r t}+\frac{r^{2}}{4} \arccos \frac{4 t^{2}-3 r^{2}}{4 r t}\right.\right. & \\
\left.\left.-\frac{1}{2} \sqrt{4 r^{2} t^{2}-\left(\frac{3}{4} r^{2}+t^{2}\right)^{2}}\right\}\right], & \frac{r}{2}<t \leq \frac{3}{2} r \\
0, & t>\frac{3}{2} r .
\end{array}\right.
$$

The probability of making the repeated round trip for all trips is given by Eq. (5).

Finally, we assume that fuel is available at neither origin $O$ nor destination $D$. If $t \leq r$, to make the round trip, the intersection of the two circles centered at $O$ and $D$ with radius $r / 2$, as depicted in Fig. 2c, must contain at least one station. The probability of making the repeated round trip for a trip of length $t$ was obtained by Miyagawa ${ }^{12)}$ as

$$
p(t)= \begin{cases}1-\exp \left\{-\frac{n}{S}\left(\frac{r^{2}}{2} \arccos \frac{t}{r}-\frac{t}{2} \sqrt{r^{2}-t^{2}}\right)\right\}, & t \leq r, \\ 0, & t>r .\end{cases}
$$

The probability of making the repeated round trip for all trips is given by Eq. (7).

\section{$5 \quad$ Numerical example}

Consider first a line segment of length $a$. Origins and destinations are assumed to be uniformly and randomly distributed within the line segment. The uniform demand serves as a basis for further analysis with actual travel demand. More realistic travel demand 
can be incorporated by using spatial interaction models. ${ }^{24)}$ The trip length distribution was derived by Koshizuka ${ }^{25)}$ as

$$
f(t)=\frac{2}{a^{2}}(a-t), \quad 0 \leq t \leq a,
$$

which is shown in Fig. 3. Using Eqs. (3), (5), and (7), we can calculate the sufficient number of stations for the three cases of the refueling availability: (a) fuel is available at both origin and destination; (b) fuel is available at either origin or destination; (c) fuel is available at neither origin nor destination. The number of stations required to achieve $P \geq \alpha$ for $a=400$ and $r=100,200$ is shown in Table 1. It can be seen that the required number of stations increases with the target probability $\alpha$ and decreases with the vehicle range $r$. To achieve a high probability (e.g., $\alpha=0.6$ for $r=100(b)$ ), many stations are required because stations are randomly distributed. It follows that, to achieve a high level of service, stations must be optimally located. Note that the effect of the refueling availability at origin and destination is significant. If fuel is available at both origin and destination, no station is needed to achieve $P \leq 0.4$ for $r=100$ and $P \leq 0.7$ for $r=200$. If fuel is available at neither origin nor destination, it is impossible to achieve $P \geq 0.5$ for $r=100$ and $P \geq 0.8$ for $r=200$.

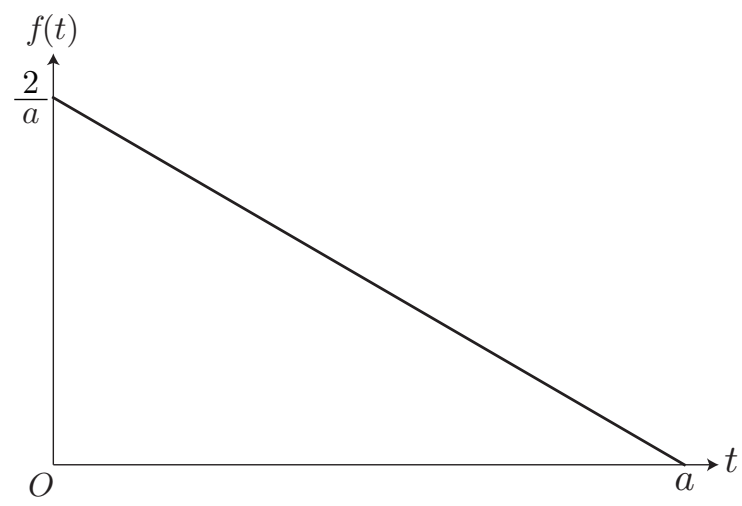

Figure 3. Trip length distribution in a line segment.

Table 1. Number of stations required to achieve $P \geq \alpha$.

\begin{tabular}{rrrrrrrrrrr}
\hline & $\alpha$ & 0.1 & 0.2 & 0.3 & 0.4 & 0.5 & 0.6 & 0.7 & 0.8 & 0.9 \\
\hline \hline$r=100$ & (a) & 0 & 0 & 0 & 0 & 2 & 7 & 22 & - & - \\
& (b) & 0 & 0 & 2 & 5 & 13 & 135 & - & - & - \\
& (c) & 3 & 6 & 12 & 42 & - & - & - & - & - \\
\hline$r=200$ & (a) & 0 & 0 & 0 & 0 & 0 & 0 & 0 & 1 & 3 \\
& (b) & 0 & 0 & 0 & 0 & 1 & 2 & 3 & 6 & 17 \\
& (c) & 1 & 2 & 2 & 4 & 5 & 9 & 22 & - & - \\
\hline
\end{tabular}

"-" means impossible.

Consider next a circle with radius $R$. Origins and destinations are assumed to be uniformly and randomly distributed within the circle. The trip length distribution was derived by Garwood and Tanner ${ }^{26)}$ as

$$
f(t)=\frac{4 t}{\pi R^{2}} \arccos \frac{t}{2 R}-\frac{t^{2}}{\pi R^{4}} \sqrt{4 R^{2}-t^{2}}, \quad 0 \leq t \leq 2 R,
$$


which is shown in Fig. 4. The number of stations required to achieve $P \geq \alpha$ for $R=200$ and $r=100,200$ is shown in Table 2. If fuel is available at both origin and destination, no station is needed to achieve $P=0.1$ for $r=100$ and $P \leq 0.5$ for $r=200$. If fuel is available at neither origin nor destination, it is impossible to achieve $P \geq 0.2$ for $r=100$ and $P \geq 0.6$ for $r=200$.

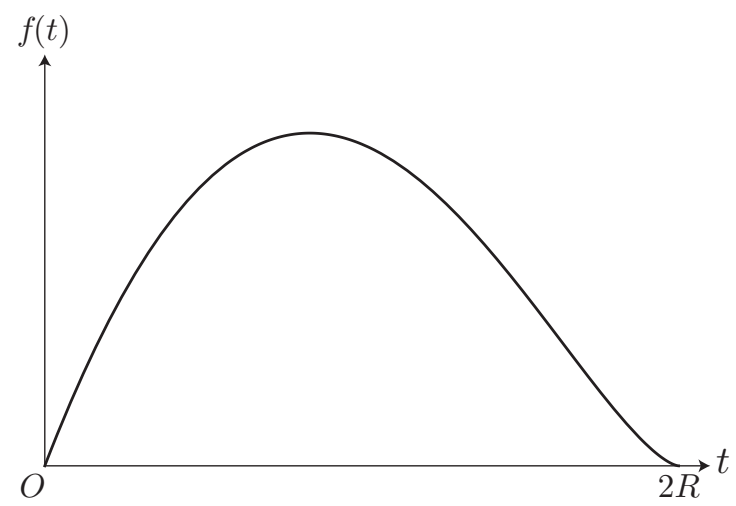

Figure 4. Trip length distribution in a circle.

Table 2. Number of stations required to achieve $P \geq \alpha$.

\begin{tabular}{rrrrrrrrrrr}
\hline & $\alpha$ & 0.1 & 0.2 & 0.3 & 0.4 & 0.5 & 0.6 & 0.7 & 0.8 & 0.9 \\
\hline \hline$r=100$ & (a) & 0 & 1 & 9 & 25 & 82 & - & - & - & - \\
& (b) & 6 & 28 & 100 & - & - & - & - & - & - \\
& (c) & 59 & - & - & - & - & - & - & - & - \\
\hline$r=200$ & (a) & 0 & 0 & 0 & 0 & 0 & 1 & 2 & 4 & 8 \\
& (b) & 0 & 1 & 2 & 3 & 6 & 9 & 15 & 32 & 2823 \\
& (c) & 3 & 7 & 13 & 27 & 82 & - & - & - & - \\
\hline "_" means impossible. & & & & & & &
\end{tabular}

\section{Conclusions}

This paper has developed a model for determining the sufficient number of stations for alternative fuel vehicles. The service level is represented as the probability that the vehicle can make the repeated round trip between randomly selected origin and destination. The model explicitly incorporates both flow demand and the trip length distribution, and will thus give a more appropriate framework for estimating the sufficient number of stations.

The model assists strategic decisions about the design of alternative fuel stations. The analytical expressions for the probability demonstrate how the number of stations, the trip length, the vehicle range, and the refueling availability at origin and destination affect the service level of stations. Note that finding these relationships by using discrete network models requires computation of the number of origin-destination pairs that can make the round trip for each combination of the parameters. The relationships help policy makers to estimate the number of stations required to achieve a certain level of service. The estimated number of stations can be used as an input in location models of alternative 
fuel stations. Comparing the effects on the service level is also useful to evaluate the effect of policies to support infrastructure development.

The present model focuses on whether the vehicle can make the round trip between origin and destination. Refueling demand, however, generally decreases with the deviation distance from the shortest path, as discussed by Miyagawa. ${ }^{27)}$ Future research should evaluate the service level considering the deviation distance to visit a station.

\section{Acknowledgements}

This research was supported by JSPS Grant-in-Aid for Scientific Research. I am grateful to Osamu Kurita and anonymous reviewers for their helpful comments and suggestions.

\section{References}

1) Greene, D.L. Survey evidence on the importance of fuel availability to the choice of alternative fuels and vehicles. Energy Studies Review, 1996, 8, 215-23.

2) Melaina, M.W. Initiating hydrogen infrastructures: Preliminary analysis of a sufficient number of initial hydrogen stations in the US. International Journal of Hydrogen Energy, 2003, 28, 743-755.

3) Melaina, M. and Bremson, J. Refueling availability for alternative fuel vehicle markets: Sufficient urban station coverage. Energy Policy, 2008, 36, 3233-3241.

4) Nicholas, M.A., Handy, S.L., and Sperling, D. Using geographic information systems to evaluate siting and networks of hydrogen stations. Transportation Research Record, 2004, 1880, 126-134.

5) Nicholas, M.A. and Ogden, J. Detailed analysis of urban station siting for California hydrogen highway network. Transportation Research Record, 2006, 1983, 121-128.

6) Bersani, C., Minciardi, R., Sacile, R., and Trasforini, E. Network planning of fuelling service stations in a near-term competitive scenario of the hydrogen economy. SocioEconomic Planning Sciences, 2009, 43, 55-71.

7) Hodgson, M.J. The location of public facilities intermediate to the journey to work. European Journal of Operational Research, 1981, 6, 199-204.

8) Berman, O., Larson, R.C., and Fouska, N. Optimal location of discretionary service facilities. Transportation Science, 1992, 26, 201-211.

9) Zeng, W., Castillo, I., and Hodgson, M.J. A generalized model for locating facilities on a network with flow-based demand. Networks and Spatial Economics, 2010, 10, $579-611$.

10) Nicholas, M.A. Driving demand: What can gasoline refueling patterns tell us about planning an alternative fuel network? Journal of Transport Geography, 2010, 18, 738-749. 
11) Kelley, S. and Kuby, M. On the way or around the corner? Observed refueling choices of alternative-fuel drivers in Southern California. Journal of Transport Geography, 2013, 33, 258-267.

12) Miyagawa, M. Density of alternative fuel stations and refueling availability. International Journal of Hydrogen Energy, 2013, 38, 12438-12445.

13) Honma, Y. and Toriumi, S. Model analysis of electric vehicle charging infrastructure development on highways -An approximation of the required scale of electric vehicle charging facilities-. FORMA, 2014, 29, 41-50.

14) Kuby, M. and Lim, S. The flow-refueling location problem for alternative-fuel vehicles. Socio-Economic Planning Sciences, 2005, 39, 125-145.

15) Kuby, M. and Lim, S. Location of alternative-fuel stations using the flow-refueling location model and dispersion of candidate sites on arcs. Networks and Spatial Economics, 2007, 7, 129-152.

16) Kuby, M., Lines, L., Schultz, R., Xie, Z., Kim, J.-G., and Lim, S. Optimization of hydrogen stations in Florida using the flow-refueling location model. International Journal of Hydrogen Energy, 2009, 34, 6045-6064.

17) Upchurch, C., Kuby, M., and Lim, S. A model for location of capacitated alternativefuel stations. Geographical Analysis, 2009, 41, 85-106.

18) Kim, J.-G. and Kuby, M. The deviation-flow refueling location model for optimizing a network of refueling stations. International Journal of Hydrogen Energy, 2012, 37, $5406-5420$.

19) Capar, I., Kuby, M., Leon, V.J., and Tsai, Y.-J. An arc cover-path-cover formulation and strategic analysis of alternative-fuel station locations. European Journal of Operational Research, 2013, 227, 142-151.

20) Larson, R.C. and Odoni, A.R. Urban Operations Research. Prentice Hall, Englewood Cliffs, NJ, 1981.

21) Sadahiro, Y. Spatiotemporal analysis of the distribution of urban facilities in terms of accessibility. Papers in Regional Science, 2005, 84, 61-84.

22) Miyagawa, M. Distribution of the sum of distances to the first and second nearest facilities. Geographical Analysis, 2014, 46, 321-333.

23) Clark, P.J. and Evans, F.C. Distance to nearest neighbor as a measure of spatial relationships in populations. Ecology, 1954, 35, 85-90.

24) Roy, J.R. Spatial Interaction Modelling, Springer-Verlag, Berlin, 2010.

25) Koshizuka, T. Comparison between low and high buildings with respect to travel distance. Journal of the City Planning Institute of Japan, 1996, 31, 31-36 (in Japanese).

26) Garwood, F. and Tanner, J.C. Note 2800: On note 2754-A repeated integral. Mathematical Gazette, 1958, 42, 292-293. 
Urban and Regional Planning Review

Vol. 3, 2016

27) Miyagawa, M. Distribution of deviation distance to alternative fuel stations. American Journal of Operations Research, 2013, 3, 363-368. 